Pacific Journal of Mathematics

TOPOLOGICAL REALIZATION OF EQUIVARIANT 


\title{
TOPOLOGICAL REALIZATION OF EQUIVARIANT INTERSECTION FORMS
}

\author{
Steven H. Weintraub
}

\begin{abstract}
Suppose $\varphi: L \otimes L \rightarrow Z$ is the equivariant intersection form of a highly-connected manifold admitting a $\boldsymbol{Z}_{p}$-action, $p$ an odd prime, so in particular $L$ is an integral representation of $Z_{p}$. We first derive conditions on $L$. Then we show that if $\varphi$ is any even unimodular form on an $L$ satisfying these conditions, there is a highly-connected manifold $M$ admitting a piecewiselinear $Z_{p}$-action having a form Witt-equivalent to $\varphi$ as its intersection form. We also prove the analogous statement for torsion linking forms of odd-dimensional manifolds. Finally, we consider the smoothing question for the actions we construct.
\end{abstract}

This paper may be considered a sequel to [9], although our emphasis here has shifted somewhat. (We are now more concerned with the forms we realize than with the manifolds we realize them with.) Let us recall one of the principal results of [9].

THEOREM. Let $\varphi: \boldsymbol{Z}^{m} \otimes \boldsymbol{Z}^{m} \rightarrow \boldsymbol{Z}$ be any even, symmetric, unimodular bilinear form. Let $\boldsymbol{Z}_{n}$ denote the cyclic group of order $n$.

Then for any integer $n$ and any $k \geqq 2$ there exists a semi-free $\boldsymbol{Z}_{n}$-action on a $(2 k-1)$-connected $4 k$-manifold $M$, with isolated fixed points, having $\boldsymbol{Z}_{n}$ act trivially on homology, and with $\varphi$ the intersection form on $M$.

This theorem may be regarded as a realization theorem for equivariant intersection forms, where the representation of $Z_{n}$ on $H_{2 k}(M)$ is the trivial one. We are then led to consider the question of representing arbitrary intersection forms. That is, suppose $L$ is an integral $\boldsymbol{Z}_{n}$-representation space and $\varphi: L \otimes L \rightarrow \boldsymbol{Z}$ is a $\boldsymbol{Z}_{n}$-equivariant, even, symmetric, unimodular bilinear form. We ask when does there exist a highly-connected $4 k$-manifold $M$ admitting a $\boldsymbol{Z}_{n^{-}}$ action with $\varphi$ the intersection form on $H_{2 l}(M)$ and $H_{2 k}(M)=L$ as a $\boldsymbol{Z}_{n}$-space.

Also, suppose that $\bar{\varphi}: \bar{L} \otimes \bar{L} \rightarrow Q / \boldsymbol{Z}$ is a $\boldsymbol{Z}_{n}$-equivariant bilinear form on a torsion module $\bar{L}$. We ask when can $\bar{\varphi}$ arise as the torsion linking form on the highly-connected boundary of a highly-connected $4 k$-manifold with a $\boldsymbol{Z}_{n}$-action.

Integral representations are rather difficult to study. In fact, if $n$ is divisible by the cube of a prime, there are infinitely many 
distinct indecomposable integral $\boldsymbol{Z}_{n}$-representations (see [5, II]). Thus, in order to enable us to solve the algebraic problems involved, we shall restrict our attention to $\boldsymbol{Z}_{p}$-actions, where $p$ is an odd prime. (In a future paper, we hope to present examples of actions of arbitrary cyclic groups.) In this case, we know (see [4, §74]) that any integral representation $L$ of $\boldsymbol{Z}_{p}$ can be written as a direct sum of indecomposable (although not irreducible) representations

$$
L=m T \oplus n A \oplus q R \oplus E
$$

where $m, n$, and $q$ are multiplicites and $T, A, R$, and $E$ are as follows:

$T=$ the trivial representation

$A=$ the representation given by the action of $\xi$ on $Z[\xi]$, where $\xi=\exp (2 \pi i / p)$ (of rank $p-1$ )

$R=$ the regular representation

$E=$ a representation arising from a nonprincipal ideal $I$ in $Z[\xi]$, there being two possibilities for each such ideal $I$, one being $I$ itself and the other being $R \otimes_{A} I$ (so for $p<23, E$ cannot occur).

(We shall use the notation in $\left(^{*}\right)$ throughout.)

We first observe

THEOREM 1.6. If $\varphi: L \otimes L \rightarrow Z$ is the equivariant intersection form of a $(2 k-1)$-connected 4k-manifold with boundary admitting a $\boldsymbol{Z}_{p}$-action, then $E$ does not appear. If the manifold is closed, $n$ is even. Also, if $\phi$ is an even form, $m \equiv q \bmod 2$.

The first part of this theorem follows from a result of Swan, while the second part is due to Conner. The third part follows trivially as an even form must have even rank. The reader may conjecture, however, that, in fact, $m$ and $q$ must both be even, but this conjecture is false, and we present counterexamples below (see 1.7).

We also observe

COROLLARY 1.3. Let $\varphi: L \otimes L \rightarrow Z$ be a $\boldsymbol{Z}_{p}$-equivariant, symmetric, bilinear (not necessarily unimodular) form, where $L=n A$, for some $n$. Then $\varphi$ is an even form.

Our main result are realization theorems. We show that any candidate for an intersection form can be realized, at least stably. To be precise, 
THEOREM 2.4. Let $\varphi: L \otimes L \rightarrow Z$ be as in the conclusion to Theorem 1.6 for a closed manifold.

Then, for any $k \geqq 2$, there is a $(2 k-1)$-connected $4 k$-manifold $M$ admitting a semi-free $Z_{p}$-action with fixed-point set $F$ a union of isolated points and with the equivariant intersection form on $M$ isomorphic to $\varphi \oplus r \varepsilon$ for $r$ sufficiently large, where $\varepsilon$ is a hyperbolic plane (i.e., the form $\left(\begin{array}{ll}0 & 1 \\ 1 & 0\end{array}\right)$ with trivial $\boldsymbol{Z}_{p}$-action).

In order to state our result for the bounded case, we must first make a definition.

Definition. Let $\varphi: L \otimes L \rightarrow Z$ be a bilinear form on the $\boldsymbol{Z}$-free $\boldsymbol{Z}_{n}$-module $L$ with $\operatorname{det}(\phi) \neq 0$.

Let $L^{\prime}$ be the dual lattice to $L$ in

$$
L \otimes Q \text {, i.e., } L^{\prime}=\left\{l^{\prime} \in L \otimes Q \mid \phi\left(l^{\prime}, l\right) \in Z \forall l \in L\right\}
$$

where $\varphi$ extends to $L \otimes Q$ as $\varphi \otimes 1$. (The condition $\operatorname{det}(\varphi) \neq 0$ is equivalent to the condition that $\varphi \otimes 1$ be nonsingular.)

Then $\bar{\varphi}: \bar{L} \otimes \bar{L} \rightarrow Q / Z$ defined by $\bar{L}=L^{\prime} / L, \varphi\left(l_{1}, l_{2}\right)=\varphi\left(l_{1}, l_{2}\right) \bmod \boldsymbol{Z}$ is the geometric boundary of $\phi$, denoted $\partial(\phi)$.

In the case that $\phi$ is the intersection form on a highly-connected manifold with boundary, then $\partial(\varphi)$ is the linking form on its boundary.

THEOREM 3.2. Let $\bar{\varphi}: \bar{L} \otimes \bar{L} \rightarrow Q / Z$ be a $\boldsymbol{Z}_{p}$-equivariant $\varepsilon$-symmetric bilinear form, such that $\bar{\phi}=\partial(\phi)$ for some $\phi$ satisfying the conclusion to Theorem 1.6. Then there is a $(k-1)$-connected $2 k$ manifold $M$ with $(k-2)-c o n n e c t e d$ boundary such that the linking form on $\partial M$ is Witt-equivalent to $\bar{\varphi}$. ( $k$ is even if $\phi$ is symmetric and odd if $\varphi$ is skew-symmetric).

Returning to the closed case, from Smith theory, we know that $F$ must consist of exactly $2+m-n+2 r$ fixed points. We can also give some conditions under which $\varphi$ can be realized exactly. (Indeed, we must confess that the need to stabilize may be merely an artifact of our construction.)

Let us remark on one difference between the situation here and that in [9]. That is, in the case where $\boldsymbol{Z}_{p}$ acts trivially on homology there is a basis of $H_{2 k}(M)$ consisting of invariant spheres. However, when $n>0$ (regardless of $q$ ), the subspace of invariant vectors of $H_{2 k}(M)$ no longer has a basis of invariant spheres. Essentially, this phenomenon arises as an invariant sphere must contain a pair of fixed points, and its homology class is partially determined by the fixed points it contains. If $n=0$, the number of fixed points exceeds 
the dimension of fixed vectors by 2 , but from the formula above, if $n>0$, this is no longer true, so when we try to find a basis, we find there are not enough fixed points to go around.

Now we turn our attention to skew-symmetric forms $\varphi$, i.e., to the case of actions on $(4 k+2)$-manifolds. In the case where $\boldsymbol{Z}_{p}$ acts trivially on homology there is little to say, as from Smith theory there can never be an action with isolated fixed points (and even a free action can only occur on a manifold with the cohomology ring of $\left.S^{2 k+1} \times S^{2 k+1}\right)$, and even neglecting the question of a fixed-point set any form $\varphi$ must be trivial and so can be realized by an obvious action on $S^{2 k+1} \times S^{2 k+1} \# \cdots \# S^{2 k+1} \times S^{2 k+1}$, taking the equivariant connected sum along the (positive-dimensional) fixed-point set. Once we allow $\boldsymbol{Z}_{p}$ to act nontrivially, however, we get an interesting situation, as then $\varphi$ need not be trivial. Here our result is somewhat weaker than in the symmetric case, as we must resort to a more violent form of stabilization. (In the skew-symmetric case $E$ still cannot appear, but $n$ need not be even, while $q$ must be.) We have

THEOREM 2.7. Let $\varphi: L \otimes L \rightarrow Z$ be a skew-symmetric unimodular bilinear form, where $L=n A \oplus q R$ (so that the trivial representation does not appear).

Then, for any $k \geqq 1$, there is a 2k-connected $(4 k+2)$-manifold $M$ admitting a semi-free $\boldsymbol{Z}_{p}$-action with fixed-point set $F$ a union of isolated points and with the equivariant intersection form on $M$ isomorphic to $\varphi$.

Here Smith theory dictates that there must be $2+n$ fixed points.

This result is the analogue of the result in the symmetric case as any unimodular skew-symmetric form $\varphi$ is Witt equivalent to a form in which the trivial representation does not appear. (Witt equivalence is the equivalence relation obtained by taking the Grothendieck ring of forms, i.e., it is the algebraic relation engendered by the topological relation of cobordism splitting all short exact sequences.) In fact, up to Witt equivalence we can eliminate the copies of the regular action, but that drastic a step we need not take.

Our constructions of actions are done with an eye toward closed manifolds, but actually proceed by first constructing manifolds-withboundary, and then filling in the boundary. Thus the results on actions on manifolds-with-boundary, which we derive in $\S 3$, fall out with little extra work (all of it spent in identifying forms). However, if one just needed the results in the bounded case the construction could be considerably simplified, as much care is taken to insure the actions constructed can be extended to closed manifolds when necessary (but it does not seem worthwhile to give a separate proof). 
All of the actions we construct are smooth except possibly at one fixed point. We can apply the G-Signature theorem to decide when these actions are (locally) smooth. In this regard, the situation when $\boldsymbol{Z}_{p}$ acts nontrivially on homology differs markedly from the case where $\boldsymbol{Z}_{p}$ acts trivially. Recall that in the latter case we showed (in [9]) that if the manifold $M$ has nonzero index, in order for the action to be smooth the dimension of $M$ must be divisible by $2 p-2$ and in the normal representation to the fixed-point set (which is the same at each fixed point) each eigenvalue of $\boldsymbol{Z}_{p}$ must appear with the same multiplicity. Once we allow the $\boldsymbol{Z}_{p}$-action on $H_{*}(M)$ to be nontrivial, however, we may obtain (locally) smooth actions in any dimension, with a different normal representation. Examples of these are given in $\S 4$.

The author would like to thank Pierre Conner for numerous helpful conversations.

1. Algebraic preliminaries. In this section we determine the explicit form of equivariant bilinear forms, which we shall need in order to construct manifolds in $\S 2$. We also prove Theorem 1.6, which eliminates many candidates for intersection forms. Also, for the convenience of the reader we explicitly give conditions on the entries of intersection matrices in order that they be equivariant.

LEMma 1.1. Suppose $\rho$ is a $\boldsymbol{Z}_{p}$-equivariant symmetric bilinear form on the integral representation space $R$. Let $H=\left(h_{i, j}\right)$ be the matrix of $\varphi$ with respect to a basis $x, t x, \cdots$. Then for $j \geqq i, h_{i, j}=$ $h_{i, j-i+1}$. Also, for $i=2, \cdots, p, h_{1, i}=h_{1, p+2-i}$.

Proof. By equivariance, $h_{1, i}=h_{2, i+1}=\cdots=h_{1+p-i, p}=h_{2+p-i, 1}=$ $h_{1, p+2-i}$.

LEMma 1.2. Suppose $\varphi$ is a $\boldsymbol{Z}_{p}$-equivariant symmetric bilinear form on the integral representation space $A$. Let $H=\left(h_{i, j}\right)$ be the matrix of $\phi$ with respect to a basis $x, t x, \cdots$. Then, for $j \geqq i, h_{i, j}=$ $h_{1, j-i+1}$. Also, for $i=3, \cdots, p-1, h_{1, i}=h_{1, p+2-i}$.

Furthermore, $h_{1,1}+2 \Sigma_{i=2}^{(p+1) / 2} h_{1, i}=0$.

Proof. If $A$ has a basis $x_{1}, \cdots, x_{p-1}, \varphi\left(x_{i}, x_{j}\right)=h_{\imath, j}$. The action of $\boldsymbol{Z}_{p}$ is given by $x_{1} \rightarrow x_{2}, x_{2} \longrightarrow x_{3}, \cdots, x_{p-2} \longrightarrow x_{p-1}, x_{p-1} \longrightarrow-\left(x_{1}+x_{2}+\cdots+x_{p-1}\right)$.

That $h_{i, j}=h_{1, j-i+1}$ is clear as above.

Now consider 


$$
\begin{aligned}
h_{1,(p+1) / 2}= & \varphi\left(x_{1}, x_{(p+1) / 2}\right)=\varphi\left(x_{(p-1) / 2}, x_{(p-1)}\right) \\
= & \varphi\left(x_{(p+1) / 2},-\left(\sum_{i=1}^{p-1} x_{i}\right)\right) \\
= & -\varphi\left(x_{(p+1) / 2}, \Sigma\left(_{i=2}^{p-1) / 2} x_{i}\right)-\varphi\left(x_{(p+1) / 2}, x_{(p+1) / 2}\right)\right. \\
& -\varphi\left(x_{(p+1) / 2}, \sum_{i=(p+3) / 2}^{p-1} x_{i}\right)-\varphi\left(x_{(p+1) / 2}, x_{1}\right) \\
= & -2 \sum_{i=2}^{(p-1) / 2} \varphi\left(x_{1}, x_{i}\right)-\varphi\left(x_{1}, x_{1}\right)-\varphi\left(x_{1}, x_{(p+1) / 2}\right) \\
= & -2 \sum_{i=2}^{(p-1) / 2} h_{1, i}-h_{1,1}-h_{1,(p+1) / 2}
\end{aligned}
$$

yielding the desired relation.

As for the first part of the lemma, let us first prove $h_{1,(p+1) / 2}=$ $h_{1,(p+3) / 2}$.

$$
\begin{aligned}
h_{1,(p+3) / 2}= & \varphi\left(x_{1}, x_{(p+3) / 2}\right)=\varphi\left(x_{(p-3) / 2}, x_{p-1}\right) \\
= & \varphi\left(x_{(p-1) / 2},-\sum_{i=1}^{p-1} x_{i}\right) \\
= & -\sum_{i=1}^{(p-3) / 2} \varphi\left(x_{(p-1) / 2}, x_{i}\right)-\varphi\left(x_{(p-1) / 2}, x_{(p-1) / 2}\right) \\
& -\sum_{i=(p+1) / 2}^{p-2} \varphi\left(x_{(p-1) / 2}, x_{i}\right)-\varphi\left(x_{(p-1) / 2}, x_{(p-1)}\right) \\
= & -2 \sum_{i=2}^{(p-1) / 2} h_{1, i}-h_{1,1}-h_{1,(p+1) / 2}
\end{aligned}
$$

and in light of the relation just proved, we must have $h_{1,(p+1) / 2}=$ $h_{1,(p+3) / 2}$. The remaining cases are proved by downward induction on $i$, by essentially the same argument.

COROLLARY 1.3. Let $\varphi: L \otimes L \rightarrow Z$ be a $\boldsymbol{Z}_{p}$-equivariant, symmetric, bilinear form, where $L$ is isomorphic to $n A$, for some value of $n$.

Then $\varphi$ is an even form.

Corollary 1.4. Let $\varphi: A \otimes A \rightarrow Z$ be a $\boldsymbol{Z}_{p}$-equivariant, symmetric bilinear form. Then $p \mid \operatorname{det}(\varphi)$.

Proof. If $H=\left(h_{i, j}\right)$ is the matrix of $\varphi$, the relation in Lemma 1.2 imply that if we multiply the vector $(1,2, \cdots, p-1)$ by $H$ the result is $0 \bmod p$.

We now turn to the determination of the off-diagonal blocks of a matrix $H$ for a form $\varphi: L \otimes L \rightarrow Z$.

First note that $T$ and $A$ are orthogonal, as they can be written as a sum of distinct irreducibles over the rationals, so a block corresponding to such a pair must be zero, while if $t$ generates $T$ and $x_{1}, \cdots, x_{p}$ generate $R, \varphi\left(t, x_{1}\right)=\cdots=\varphi\left(t, x_{p}\right)$.

As for the remaining cases, they are most conveniently summarized as follows:

LEMmA 1.5. Let $L=2 A \oplus 2 R$ and let $\varphi: L \otimes L \rightarrow Z$ be a symmetric bilinear form with matrix $H$ (with respect to the obvious basis) 


$$
H=\left(\begin{array}{llll}
* & A & * & * \\
* & * & B & * \\
* & * & * & C \\
* & * & * & *
\end{array}\right)
$$

(so that $A$ is $(p-1) \times(p-1), B$ is $(p-1) \times p$, and $C$ is $p \times p)$. Then

(a) If $A=\left(a_{i, j}\right), a_{i+k, j+k}=a_{i, j}$ as long as $i+k \leqq p-1, j+k \leqq$ $p-1$, and for $k=3, \cdots, p-1, a_{1, k}=a_{p+2-k, 1}$. Furthermore, $a_{2,1}+$ $\sum_{k=1}^{p-1} a_{1, k}=0$.

(b) If $B=\left(b_{i, j}\right), b_{i+k, j+k}=b_{i, j}$ as long as $i+k \leqq p-1, j+k \leqq p$, and for $k=3, \cdots, p, b_{1, k}=b_{p+2-k, 1}$. Furthermore, $\sum_{k=1}^{p} b_{1, k}=0$.

(b) If $C=\left(c_{i, j}\right), c_{i+k, j+k}=c_{i, j}$ for all $i, j, k$, with the subscripts interpreted $\bmod p$.

Proof. Routine.

Note that each of the matrices $A, B$, and $C$ is determined by its first row. The conditions given in 1.1,1.2, and 1.5 turn out to be sufficient as well as necessary, i.e., any matrix satisfying them can occur as the matrix of a form, although not necessarily of a unimodular one.

THEOREM 1.6. If the even form $\varphi: L \otimes L \rightarrow Z$ is the equivariant form of $a(2 k-1)$-connected $4 k$-manifold $M$ with boundary with a $\boldsymbol{Z}_{p}$-action, and $L$ is written as a sum of indecomposables, $L=m T \oplus$ $n A \oplus q R \oplus E$, then $E$ does not appear. If $M$ is closed, $n$ must be even. Also, if $\varphi$ is even, $m \equiv q \bmod 2$.

Proof. The last assertion is trivial as a unimodular even form must have even rank.

For the first assertion, recall the following result of Swan:

Let $\pi$ be a finite group and let $G(Z \pi)$ be the Grothendieck group of finitely-generated $\boldsymbol{Z} \pi$ modules, and similarly for $\boldsymbol{Q} \pi$. Let $o$ be a maximal order of $\boldsymbol{Q} \pi$, and $C_{0}(0)$ its reduced projective class group. (If $\pi$ is $Z_{p}, o$ is $Z[\exp (2 \pi i / p)]$.) Then, [7, Corollary 13.2], for $\pi$ cyclic

$$
G(\boldsymbol{Z} \pi)=G(\boldsymbol{Q} \pi) \times C_{0}(0)
$$

Now, suppose $Z_{p}$ is acting simplicially on $M$. Then the equivariant simplicial chain complex $C$ of $M$ is generated by simplices which $Z_{p}$ either fixes or permutes freely. Hence the image of $C$ in $G(Z \pi)$ projects to zero in $C_{0}(0)$. But this image is exactly the same as the image of the chain complex $H$ which is the homology of $M$ (i.e., $H$ has zero differential and in dimension $i$ it is $\left.H_{i}(M)\right)$. But in our case this 
image is just $\left[H_{0}(M)\right]+\left[H_{2 k}(M)\right]+\left[H_{4 k}(M)\right]=2[T]+\left[H_{2 k}(M)\right]$. Since this class projects to 0 in $C_{0}(o)$, so must $H_{2 k}(M)$. But if $H_{2 k}(M)=$ $m T \oplus n A \oplus q R \oplus E$, this projection is zero iff $E$ does not appear.

Now let us assume $M$ is closed, so that $\phi$ is unimodular.

To show $n$ is even, let $\tau$ denote the action of a generator of $\boldsymbol{Z}_{p}$. We have the operators $\Sigma=I+\tau+\cdots+\tau^{p-1}$ and $\Delta=(1-\tau)$ on $L$, with $\Delta_{0} \Sigma=\Sigma_{0} \Delta=0$ (in fact, with $(\operatorname{ker} \Sigma)^{\perp}=\operatorname{ker} \Delta$ and $(\operatorname{ker} \Delta)^{\perp}=$ $\operatorname{ker} \Sigma)$, and $\varphi(v, \Sigma w)=\varphi(\Sigma v, w), \phi(v, \Delta w)=-\varphi(\Delta v, w)$.

We will define a nonsingular $\boldsymbol{Z}_{p}$-valued skew-symmetric bilinear form on $\operatorname{ker}(\Sigma) / \operatorname{Im}(\Delta)$. But $\operatorname{rank}_{z_{p}} \operatorname{ker}(\Sigma) / \operatorname{Im}(\Delta)=n$, the number of copies of $A$, so $n$ must be even.

For $x, y \in \operatorname{ker}(\Sigma)$, choose $z$ with $\Delta z=p y$ (which can always be done as $\operatorname{Im}(\Delta) \supset p \operatorname{ker}(\Sigma))$ and set $\bar{\beta}(x, y)=\varphi(x, z)$.

Then $\beta(x, y)=\bar{\beta}(x, y) \bmod p$ is our desired form.

1. $\beta$ is independent of the choice of $z$. For suppose $\Delta z=0$, i.e., $z$ is fixed under $\tau$. Then $\Sigma z=p z$ so $p \varphi(x, z)=\varphi(x, p z)=\varphi(x, \Sigma z)=$ $\phi(\Sigma x, z)=0$ as $x \in \operatorname{ker}(\Sigma)$.

2. $\operatorname{Im}(\Delta) \subset \operatorname{Radical}(\beta)$ so we may pass to the quotient. For suppose $y \in \operatorname{Im}(\Delta)$. Then $\exists \bar{z}$ with $\Delta \bar{z}=y$, so $\Delta p \bar{z}=p y$ and $\bar{\beta}(x, y)=$ $\varphi(x, p \bar{z}) \equiv 0 \bmod p$.

3. Radical $(\beta) \subset \operatorname{Im}(\Delta)$ so $\beta$ is nonsingular. For suppose $x \in$ Radical $(\beta)$, so that $\beta(x, y) \equiv 0$. Then $\bar{\beta}(x, y)$ is always divisible by $p, \bar{\beta}(x, y)=p \psi(y)$. As $\operatorname{ker}(\Sigma)$ is a direct summand, $\exists w$ with $\psi(y)=$ $\bar{\beta}(-w, y)$.

Then

$$
p \psi(y)=p \bar{\beta}(-w, y)=\bar{\beta}(-w, p y)=\bar{\beta}(-w, \Delta z)=\bar{\beta}(\Delta w, z)
$$

for some $z$, so $\bar{\beta}(x-\Delta w, z)=0$ for all $z \in p \operatorname{ker}(\Sigma)$, so $\bar{\beta}(x-\Delta w, z)=0$ for all $z \in \operatorname{ker}(\Sigma)$ as $\bar{\beta}$ is integer-valued and $p \operatorname{ker}(\Sigma)$ is of finite index in $\operatorname{ker}(\Sigma)$. While, if $z \in \operatorname{ker}(\Delta), \tau z=z$, so $\Sigma z=p z$ and

$$
p \bar{\beta}(x-\Delta w, z)=\bar{\beta}(x-\Delta w, \Sigma z)=\bar{\beta}(\Sigma(x-\Delta w), z)=0
$$

as $x \in \operatorname{ker}(\Sigma)$. Hence we must have $x=\Delta w$, so $x \in \operatorname{Im}(\Delta)$.

4. $\beta$ is skew-symmetric. It suffices to show $\beta(x, x)=0 \forall x$. Now $\bar{\beta}(x, x)=\varphi(x, z)$ where $\Delta z=p x$, so

$$
p \bar{\beta}(x, x)=\varphi(p x, z)=\varphi(\Delta z, z)=\varphi(z,-\Delta z)=\varphi(z,-p x)=-\varphi(p x, z) .
$$

But $\phi$ is symmetric so $\bar{\beta}(x, x)=0$.

REMARK. A similar argument applied to $L / \operatorname{ker}(\Delta) \oplus \operatorname{ker}(\Sigma)$ shows that the number of copies of the group-ring appearing must be even in the case of a unimodular skew-symmetric form $\varphi$.

Now we present an example of an even, symmetric unimodular 
form on $L=m T \oplus q R$ with $m$ and $q$ both odd.

Proposition 1.6. Let $H=\left(h_{i, j}\right)$ be the $(2 p-2) \times(2 p-2)$ matrix with entries given by

(a) $h_{i, j}=1$ if $i \neq j, 1 \leqq p+1, j \leqq p+1$,

(b) $h_{i, j}=1$ if $|i-j|=1$,

(c) $h_{i, i}=0, i=1, \cdots, p$,

(d) $h_{i, i}=2, i=p+1, \cdots, 2 p-2$.

Then $H$ is equivariant with respect to the representation $R \oplus$ $(p-2) T$ (with the obvious basis) and $\operatorname{det}(H)=1$.

Proof. Equivariance is clear from 1.1 and the remarks preceding 1.5 .

To prove $\operatorname{det}(H)=1$, let $H_{s}$ be the $s \times s$ matrix in the upper left-hand corner of $H$ (i.e., $\left.H_{s}=\left(h_{i, j}\right), i=1, \cdots, s, j=1, \cdots, s\right)$. First we claim $\operatorname{det}\left(H_{p}\right)=p-1$. To show this, add each of the first $(p-1)$ rows to the last row, so that all of the entries in the last row become equal to $p-1$, and then subtract $1 /(p-1)$ times the last row from each of the first $(p-1)$ rows, so that all of the entries in each become zero, except for the diagonal entires which all become -1 . Then $\operatorname{det}\left(H_{p}\right)=(-1)^{p-1}(p-1)=(p-1)$.

Now we claim $\operatorname{det}\left(H_{p+1}\right)=p-2$. To show this, subtract $1 /(p-1)$ times the sum of the first $p$ rows from the last row, obtaining a row which is all zero except for the last entry, which is now $2-p /(p-1)=(p-2) /(p-1)$. Then

$$
\operatorname{det}\left(H_{p+1}\right)=(p-2) /(p-1) \cdot \operatorname{det}\left(H_{p}\right)=p-2 .
$$

For $s>p+1, H_{s}$ is obtained from $H_{s-1}$ by adjoining a row and column all of whose entries are zero, except for $h_{s-1, s}=h_{s, s-1}=1$ and (possibly) $h_{s, s}$. Expanding by minors of the last row yields the formula

$$
\operatorname{det}\left(H_{s}\right)=h_{s, s} \operatorname{det}\left(H_{s-1}\right)-\operatorname{det}\left(H_{s-2}\right) .
$$

Here, $h_{s, s}=2$ for $s>p+1$. Then applying the above formula shows inductively that $\operatorname{det}\left(H_{s}\right)=(p-2)-(s-(p+1))=2 p-1-s$ so $\operatorname{det}(H)=\operatorname{det}\left(H_{2 p-2}\right)=1$.

We now compute the determinants of matrices which we will use in $\S 2$.

Lemma 1.8. Let $H_{s}=\left(h_{i, j}\right)$ be the $s \times s$ matrix given by

(a) $h_{i, j}=1, i \neq j$,

(b) $h_{i, i}=0, i=1, \cdots,[(s+1) / 2]$,

(c) $h_{i, i}=2, i=[(s+1) / 2]+1, \cdots, s$. 
Then $\operatorname{det}\left(H_{s}\right)=(-1)^{s / 2}$ for $s$ even, and $\operatorname{det}\left(H_{s}\right)=0$ for $s$ odd.

Proof. Subtract the first row from each of the other rows. Each of these rows then has a 1 in the first column, and all other entries 0 except that on the diagonal, which is -1 for the second through the $[(s+1) / 2]$-nd row and 1 for the rest. Then the second through $[(s+1) / 2]-n d$ rows to the first row and subtract the remaining rows from the first row. All the entries in the first row are now zero, except for the first, which is 0 if $s$ is odd and -1 if $s$ is even. Then $\operatorname{det}\left(H_{s}\right)=0$ for $s$ odd and $(-1)^{s / 2}$ for $s$ even (as the first $s / 2$ rows have a -1 on the diagonal and the last $s / 2$ rows $a+1)$.

Now we turn to the skew-symmetric case. First we observe that $E$ also cannot appear here-the proof of 1.6 holds unchanged in this case. On the other hand, $n$ need not be even, and we will present an example of a unimodular skew-symmetric form on $A$ shortly.

First, however, let us determine the form of an equivariant skew-symmetric bilinear form, i.e., the analogues to $1.1,1.2$, and 1.5.

LEMMA 1.9. Suppose $\varphi$ is a $\boldsymbol{Z}_{p}$-invariant skew-symmetric bilinear form on the integral representation space with matrix $H=\left(h_{i, j}\right)$. Then $h_{i, i}=0$ and $h_{i, j}=-h_{j, i}$. Furthermore,

(a) if $L=R, h_{1, i}=-h_{1, p+2-i}, 2 \leqq i \leqq p$

(b) if $L=A, h_{1, i}=-h_{1, p+2-i}, 3 \leqq i \leqq p-1$.

Proof. Routine.

Lemma 1.10. Let $L=2 A \oplus 2 R$ and let $\varphi: L \otimes L \rightarrow Z$ be a skewsymmetric bilinear form with matrix $H$ (with respect to the obvious basis)

$$
H=\left(\begin{array}{llll}
* & A & * & * \\
* & * & B & * \\
* & * & * & C \\
* & * & * & *
\end{array}\right)
$$

(so that $A$ is $(p-1) \times(p-1), B$ is $(p-1) \times p$ and $C$ is $p \times p)$. Then $A, B$, and $C$ are as in the conclusion of 1.5.

Proof. It is never necessary to change the order of the inner products in proving 1.5 so the same computation that proves that lemma in the symmetric case proves it in the skew-symmetric case.

Now for the promised example. 
Proposition 1.11. Let $H=\left(h_{i, j}\right)$ be the $(p-1) \times(p-1)$ matrix with entries given by

(a) $h_{i, j}=1$ if $j=i+1$,

(b) $h_{i, j}=-1$ if $j=i-1$,

(c) $h_{i, j}=0$ if $|i-j| \neq 1$.

Then $H$ is equivariant with respect to the representation $A$ and $\operatorname{det}(H)=1$.

Proof. That $H$ is equivariant follows from 1.9. Unimodularity is easy to check using $\left({ }^{* *}\right)$.

2. Geometric constructions. In this section we construct manifolds with given equivariant intersection forms.

All of our constructions will be performed "upstairs" rather than "downstairs," i.e., in the total space of the action rather than in the quotient. However, this will cause no problem with equivariance, as all modification will be done either on invariant (or perhaps fixed) sets, or else will be done on a set which is acted upon freely and is disjoint from all its translates, and then will be copied exactly on these translates, so as to maintain invariance under the group action.

The construction proceeds by assembling "building blocks." We begin by showing how to obtain these blocks.

Lemma 2.1. Let $\varphi: A \otimes A \rightarrow Z$ be an equivariant symmetric bilinear form.

Then, for any $k \geqq 1$, there exists a $(2 k-1)$-connected $4 k$-manifold $M$ with $(2 k-2)$-connected boundary, and a $Z_{p}$-action on $M$, such that $\varphi$ is the equivariant intersection form of $M$.

The action may be chosen so as to be free, or to have fixed-point set $F=S^{2 q-1} \times D^{2 r+1}, q, r<k$.

Proof. Let $M_{0}=S^{2 k-1} \times D^{2 k+1}$. If $M$ is embedded in the obvious way in $C^{k} \times C^{k} \times R$, let $Z_{p}$ act on $M_{0}$ by

$$
g\left(z_{1}, \cdots, z_{2 k}, x\right)=\left(\exp (2 \pi i / p) z_{1}, \cdots, \exp (2 \pi i / p) z_{k}, x\right) \text {. }
$$

Let $S^{2 k-1}=S_{0}$ be embedded in $M_{0}$ by a map $x \rightarrow(x, *)$, where * is a point moved freely by $Z_{p}$. Let $S_{1}, S_{2}, \ldots$ be its translates under $Z_{p}$. They are then mutually disjoint, and $\left[S_{0}\right]=\cdots=\left[S_{p-1}\right] \in H_{2 k-1}\left(M_{0}\right)$ is the generator.

Of course, $S_{0}$ has trivial normal bundle in $\partial M_{0}$, and so, choosing a framing of this normal bundle $\nu\left(S_{0}\right)$, we may attach a $2 k$-handle $D_{0}$ 
with core $S_{0}$ to $\partial M_{0}$. If we perform this attaching equivariantly, choosing sufficiently small tubular neighborhoods to make all the handles disjoint, and translating the framing chosen on $\nu\left(S_{0}\right)$ to obtain framings on $\mathcal{\nu}\left(S_{i}\right)$, we obtain a new manifold with $H_{2 k-1}=0, H_{2 k}=Z^{p-1}$, and in fact $H_{2 k}=A$ as a $Z_{p}$-representation lattice. Generators for $H_{2 k}$ are given by the embedded spheres $D_{i} \cup S_{i} \times I \cup-D_{i+1}, i=0, \cdots$, $p-2$. This remains true if instead of attaching along $S_{0}, \cdots, S_{p-1}$ we attach the handles along spheres homotopic to $S_{0}, \cdots, S_{p-1}$, and if the new spheres are isotopic, they will also have trivial normal bundle. (Observe that the choice of framing does not affect the resulting intersection form, as follows: Let $\bar{S}_{i}=D_{i} \cup S_{i} \times I \cup-D_{i+1}$, $i=0, \cdots, p-2$. The framing only affects the self-intersections of the $S_{i}$, which we calculate by making the $\bar{S}_{i}$ transverse to themselves. In doing this, isotopy the embedding of $\bar{S}_{i} \mid D_{i}$, and then that of $\bar{S}_{i} \mid\left(-D_{i+1}\right)$ to the translate of the new embedding of $\bar{S}_{i} \mid D_{i}$. Then we see our isotopy has the effect that the self-intersections of one of these disks will cancel those of the other, and so all the contribution to the self-intersection number comes from $\bar{S}_{i} \mid S_{i} \times I$, which is independent of the framing.)

This is in fact what we shall do, choosing the spheres correctly so as to obtain the desired intersection form.

Let $\varphi$ have matrix $H=\left(h_{i, j}\right)$. Recally from 1.2 that $H$ is determined by $h_{1,2}, h_{1,3}, \cdots, h_{1,(p+1) / 2}$ and the requirement of equivariance.

Suppose $h_{1,(p+1) / 2}=s$. We may change the embedding of $S_{0}$ in $s$ small disks, and then the embeddings of $S_{1}, \cdots, S_{p-1}$ by equivariance, so that $S_{0}$ will link each of $S_{1}, \cdots, S_{(p+1) / 2}$ exactly $s$ times, with the new $S_{0}$ isotopic to the old. Now change the embedding of $S_{0}$ in $h_{1(p-1) / 2}-h_{1,(p+1) / 2}=s^{\prime}$ small disks to link $S_{1}, \cdots, S_{(p-1) / 2} s^{\prime}$ times, changing the embeddings of the embeddings of $S_{1}, \cdots, S_{p-1}$ by equivariance, and similarly for $h_{1,(p-3) / 2}-h_{1,(p-1) / 2}$ down to $h_{1,2}-h_{1,3}$.

(One may visualize this as running tubes from $S_{0}$ to link the other spheres. We start with the longest tube. As this links with all the intervening spheres, we correct for these extra linkings with the next shortest tube, and so on till we finish with the tube linking each sphere to the next.)

When the handles are added these linkings become intersections, and the resulting manifold $M$ has the desired intersection form. (In the case when $F$ is to be nonempty, we choose an action on $M_{0}$ with the desired fixed-point set and restrict our modifications to occur in the free part of the action.)

$M$ is $(2 k-1)$-connected, as we have killed $H_{2 k-1}\left(M_{0}\right) . \quad \partial M$ is $(2 k-2)-$ connected by a Mayer-Vietoris argument as it is the union of $(2 k-2)$ connected subspaces alnng $(2 k-2)$-connected subspaces.

(Observe that the action on $M_{0}$ could have been chosen to be 
any linear representation of $\boldsymbol{Z}_{p}$. This will be of use to us in §3.)

It should be noted that the manifolds constructed above are just the "standard twisted models" of Lance [6] and what we have shown is just how to explicitly realize all possible models.

Proposition 2.2. Suppose $Z_{p}$ acts on a $(k-1)$-connected $2 k$ manifold $M$, such that $H_{k}(M)$ has a decomposition as an integral $Z_{p}$-representation $m T \oplus n A \oplus q R$.

Let $F$ be the fixed-point set of $\boldsymbol{Z}_{p}$. Then the Euler characteristic $\chi(F)=2+(-1)^{k}(m-n)$.

Proof. We have from Smith theory (see [2, III. 4.3]), (*) $\quad \chi(M)+(p-1) \chi(F)=p \chi\left(M / Z_{p}\right)$.

Let us calculate the Euler characteristic by using homology with rational coefficients. Then we also know (see [2, III. 2.4]) that $H_{*}\left(M / \boldsymbol{Z}_{p}\right)=H_{*}(M)^{z_{p}}$.

Substituting into $\left({ }^{*}\right)$, we have then

$$
2+(-1)^{k}(m+(p-1) n+p q)+(p-1) \chi(F)=p\left(+(-1)^{k}(m+q)\right)
$$

yielding the stated formula.

Let us now recall a lemma which will be of use to us. It may be found as [3, Lemma V. 2.7] where it is stated in a special case, but the proof of our statement below is identical.

LEMma 2.3. Let $M$ be a $(k-1)$-connected 2k-manifold with $(k-2)$-connected boundary $\partial M$. Let $\varphi: H_{k}(M) \otimes H_{k}(M) \rightarrow Z$ be the intersection form of $M$. Assume $k>2$.

Then $\partial M$ is a homotopy sphere if and only if $\varphi$ is unimodular.

Now we come to one of our main results.

THEOREM 2.4. Let $p$ be an odd prime and let $\varphi: L \otimes L \rightarrow Z$ by any symmetric, unimodular, even, $\boldsymbol{Z}_{p}$-equivariant bilinear form, when the lattice $L$ has a decomposition as a $\boldsymbol{Z}_{p}$-representation space $L=m T \oplus n A \oplus q R$.

Then for any $k \geqq 2$, there exists $a(2 k-1)$-connected $4 k$-manifold $M$ admitting a semi-free $Z_{p}$-action with $m-n+2 r+2$ isolated fixed points and having $\phi \oplus r \varepsilon$ as its equivariant intersection form, where $\varepsilon$ is the 2-dimensional hyperbolic plane on which $\boldsymbol{Z}_{p}$ acts trivially, for $r$ sufficiently large.

Proof. The difficult part of the construction lies in realizing 
$\varphi_{A}=\varphi \mid n A$, so we take care of that first, and then we add on the rest. In fact, we will initially have to realize $\phi_{A} \oplus n \varepsilon$.

Let $\varphi_{A}^{i}$ be $\varphi_{A}$ restricted to the $i$ th copy of $A$. Let $M_{1}, \cdots, M_{n}$ be manifolds with boundary, as in 2.1 , realizing $\varphi_{1}, \cdots, \varphi_{n}$, respectively, and with $\boldsymbol{Z}_{p}$ acting as in 2.1.

In $\partial M_{i}$ we have the invariant sphere $S^{2 k-1} \times(0, \cdots, 0,1) . \quad$ A neighborhood of this invariant sphere in $\partial M$ is equivariantly diffeomorphic to a neighborhood of the equator in $S^{2 k} \times D^{2 k}$ with the $Z_{p}$ action given by multiplying each complex coordinate by $\exp (2 \pi i / p)$, a bundle with Euler class zero, as well as to a neighborhood of the equator in the tangent bundle of $S^{2 k}$ with action induced from that on $S^{\% k}$ of multiplying each complex coordinate by $\exp (2 \pi i / p)$, a bundle with Euler two.

Attach a $2 k$-handle equivariantly, using this sphere as a core, to each of $M_{1}, \cdots, M_{n}$, choosing the attaching maps so that its homology class has self-intersection 0 on $M_{1}, \cdots, M_{n / 2}$ and self-intersection 2 on $M_{p / 2+1}, \cdots, M_{n}$. Let the results be $M_{1}^{\prime}, \cdots, M_{n}^{\prime}$.

Now each $M_{i}^{\prime}$ has the same normal representation, $g\left(z_{1}, \cdots, z_{2 k}\right)=$ $\left(\exp (2 \pi i / p) z_{1}, \cdots, \exp (2 \pi i / p) z_{k}\right)$ around its fixed point. Thus, we may take $M^{\prime}=D^{4 k} \cup M_{1}^{\prime} \cup \cdots \cup M_{n}^{\prime}$, where $D^{4 k}$ has the above $Z_{p}$-action, and each $M_{i}^{\prime}$ is identified with a linear subspace of $D^{4 k}$. Then each of these submanifolds will be transverse to each other with exactly one self-intersection, at the fixed point.

Thus at this stage we have a manifold $M^{\prime}$ with boundary realizing the intersection form, whose matrix is

$$
\left|\begin{array}{llllll}
\varphi_{A}^{1} & & & & & \\
& * & 1 & & & \\
& & \varphi_{A}^{2} & & & \\
& 1 & * & 1 & \\
& & \varphi_{A}^{3} & & \\
& & 1 & * & \\
& & & & \ddots
\end{array}\right|
$$

where ${ }^{*}=0$ or 2 . Rearranging the rows, write the intersection form as follows, with $H_{n}$ as in 1.8 .

$$
\left(\begin{array}{cccc}
\varphi_{A}^{1} & & & \\
\varphi_{A}^{2} & & \\
& \ddots & \\
& & \varphi_{A}^{n} \\
& & & H_{n}
\end{array}\right) .
$$


We may next realize the off-diagonal blocks of $\phi_{A}$ by plumbing together spheres representing the generators the appropriate number of times.

Referring to 1.5 , we see algebraically that in all such blocks intersection numbers are determined by their first row, and so we may plumb together the first sphere in the $i$ th block along the diagonal with all the spheres in the $j$ th block along the diagonal, and then equivariancew ill require us to plumb the remaining spheres in the $i$ th block with those in the $j$ th block so as to correctly obtain all of the (off-diagonal) entries in the $(i, j)$ th block. Since this occurs in the free part of the action, there is no problem with plumbing translates.

Now let us choose a subspace $T^{\prime}$ of $m T$ of dimension $n$ with $\varphi_{T}{ }^{\prime}=\varphi \mid T^{\prime}$ unimodular. If $T^{\prime}$ does not exist, we may take the direct sum of $(n / 2) \varepsilon$ with $\varphi$ to create such a subspace. Then by the proof of $[9,1.5], \varphi_{T}$, has a basis such that its matrix $H^{\prime}$ is congruent to $H_{n} \bmod p$. Realize the intersections specified by $H^{\prime}-H_{n}$ by plumbing in the free part of the action. Once again there is no problem with equivariance as each plumbing is to be done a multiple of $p$ times, since every entry in $H-H_{n}$ is divisible by $p$, so perform the plumbing at a point and each of its translates.

Now we realize $\varphi \mid T^{\prime \prime}$, where $T^{\prime \prime}$ is the orthogonal complement of $T^{\prime}$ in $T$. (This is exactly the construction in [9].) Suppose for simplicity that $\varphi_{T^{\prime \prime}}=\varphi \mid T^{\prime \prime}$ is unimodular. Equivariantly plumb $S^{2 k} \times D^{2 k}$ with the above-mentioned action to $M^{\prime}$ at the unique fixed point of $M^{\prime}$.

The manifold now constructed has intersection matrix $H_{n+1}$, as this last sphere intersects each other sphere transversely once. Now equivariantly plumb the remaining sphere of $T^{\prime \prime}$ successively using trivial bundles each to the unused fixed point of the previous one, so that each homology class has a self-intersection of zero and an intersection of zero with every other homology class except for the ones immediately preceding and following it, which it intersects once. Note that the representations at the successive fixed points differ only in sign. Inductively, it is easy to compute that the intersection matrix so obtained is unimodular. As before, since both this matrix and $\varphi_{T}$ are unimodular, there is a basis for which their matrices are congruent $\bmod p$, so do the additional plumbings in the free part of the action to get then to be identical.

If $\varphi_{T}{ }^{\prime \prime}$ is not unimodular, instead of using trivial bundles to plumb with we must use copies of the tangent bundle, with selfintersection 2. An inductive computation reveals that the resulting matrix may have any prescribed determinant $\bmod p$, providing the dimension of $T^{\prime \prime}$ is correct. If it is not, we may add more copies of $\varepsilon$ to $\rho$ to make it so, for formula $\left(^{* *}\right)$ in the proof of Proposition 
1.7 shows that as we plumb with additional bundles the determinant of the resultant intersection matrix ranges through all values $\bmod p$. Then proceed as above. Note that the representations at all the fixed points are the same.

We are now left with the task of representing the form on $q R$, but this is the easiest part.

In a disk around the hitherto unused fixed point, choose a linear subspace. Then attach an arbitrary bundle by it and its translates, thus realizing a copy of $R$ with prescribed self-intersections. One may do this for each copy. Now all other plumbing involving spheres representing elements of $R$ need to be done a multiple of $p$ times, so may be done equivariantly in the free part of the action, at a point and its translates.

Let the resulting manifold be $M^{\prime \prime}$. Now $M^{\prime \prime}$ is $(2 k-1)$-connected except for the presence of a fundamental group.

$\pi_{1}\left(M^{\prime \prime}\right)$ is a free group, however, with a generator arising each time a plumbing is done on an already connected manifold. But all such plumbings here were done equivariantly in the free part of the action, so $\pi_{1}$ may be killed by equivariant surgeries on the generators of $\pi_{1}$. Let the result be $M^{\prime \prime \prime}$.

Now $M^{\prime \prime \prime}$ is highly-connected, as is its boundary, and $\varphi \oplus r \varepsilon$ is unimodular, so by $2.3, \partial M$ is a homotopy sphere.

Let $M=M^{\prime \prime \prime} \bigcup_{\sigma M} D^{4 k}$ and extend the action to $D^{4 k}$ by coning. Then $M$ is as required.

By examining the proof we can see where stabilization was required. Thus we may observe:

Corollary 2.5. Let $\varphi$ be as in 2.4. Suppose that $L=m T \oplus q R$. Then $\varnothing$ may be realized as in 2.4 .

Alternatively, if $n \neq 0$, suppose that $\varphi_{T}$ is unimodular. Suppose $T$ has a subspace $T^{\prime}$ of rank $2 n$ with $\varphi_{T^{\prime}}$ unimodular. Then $\varnothing$ may be realized as in 2.4 .

Note, of course, that under the assumptions of the second part of the corollary, $\varphi$ actually splits, i.e., with respect to some basis

$$
\varphi=\varphi_{T^{\prime}} \oplus \varphi_{T^{\prime \prime}} \oplus \phi^{\prime \prime} .
$$

In the case where $m$ and $n$ are both zero, this realization theorem is already known from a different perspective. It is an easy consequence of the realizability of Wall group elements.

Now for the skew-symmetric case. First, the analogue of 2.1.

Lemma 2.6. Let $\varphi: A \otimes A \rightarrow Z$ be an equivariant, skew-symmetric bilinear form. 
Then, for any $k \geqq 1$, there exists a 2k-connected $(4 k+2)$-manifold $M$ with $(2 k-1)$-connected boundary, and a $Z_{p}$-action on $M$, such that $\varphi$ is the intersection form of $M$.

The action may be chosen to have fixed-point set $S^{2 q} \times$ $D^{2 r+2}, q, r<k$.

In particular, $F$ may be chosen to be $S^{0}$, contained in the interior of $M$.

Proof. Let $M_{0}=S^{2 k} \times D^{4 k+2}$ and choose a linear action on $M_{0}$ to obtain $F$ as required.

Note by 1.9 that $H$, the matrix of $\varphi$, is determined by $h_{1,2}, \cdots$, $h_{1,(p+1) / 2}$ in this case also, so we may proceed exactly as in 2.1. (Our constructions are again done away from $F$.)

THEOREM 2.7. Let $p$ be an odd prime and let $\varphi: L \otimes L \rightarrow Z$ be any skew-symmetric, unimodular, $\boldsymbol{Z}_{p}$-equivariant bilinear form, where the lattice $L$ has a decomposition as a $\boldsymbol{Z}_{p}$-representation space $L=n A \oplus q R$.

Then, for any $k \geqq 1$, there exists a $2 k$-connected $(4 k+2)$-manifold $M$ admitting a semi-free $\boldsymbol{Z}_{p}$-action with $N+2$ isolated fixed points and having $\phi$ as its equivariant intersection form.

Proof. If $L=n A \oplus q R$, let $\varphi_{A}=L \mid n A$, and then let $\phi_{A}^{i}$ be the restriction of $\phi_{A}$ to the $i$ th copy of $A$.

Construct manifolds $M_{i}$ as in 2.6 with fixed-point set $S^{\circ}$ and intersection form $\phi_{i}$ on $M_{i}$. Choose the $\boldsymbol{Z}_{p}$-action so that the pairs of representations on each fixed-point set (the representations at the two points of $S^{0}$ differing from each other only in sign) are the same on each $M_{i}$.

Now let $M^{\prime}=M_{1} \cup M_{2} \cup \cdots \cup M_{n}$, where $M_{i}$ is identified with $M_{i+1}$ equivariantly at a fixed point, and $M_{i+1}$ with $M_{i+2}$ equivariantly at a fixed point of $M_{i+2}$ and the other fixed point of $M_{i}$.

The intersection form on $M^{\prime}$ is given by the diagonal blocks of $\varphi_{A}$.

Now complete the construction as in the symmetric case, using the unused fixed point of $M_{n}$ to attach $p$ copies of $S^{2 k+1} \times D^{2 k+1}$ which are permuted cyclically by the action of $\boldsymbol{Z}_{p}$, performing appropriate plumbings to realize the off-diagonal blocks, and then killing the fundamental group.

3. Linking forms. The objective of this section is to prove Theorem 3.2. All of the geometry has already been done; what remains is the algebra. 
DeFinition 3.1. Let $\varphi: L \otimes L \rightarrow Z$ be a bilinear form on the $Z$-free $Z_{n}$-module $L$ with $\operatorname{det}(\varphi) \neq 0$.

Let $L^{\prime}$ be the dual lattice to $L$ in $L \otimes Q$, i.e., $L^{\prime}=\left\{l^{\prime} \in L \otimes\right.$ $\left.Q \mid \varphi\left(l^{\prime}, l\right) \in \boldsymbol{Z} \forall l \in L\right\}$.

Then $\bar{\varphi}: \bar{L} \otimes \bar{L} \rightarrow Q / Z$ defined by $\bar{L}=L^{\prime} / L, \bar{\varphi}\left(l_{1}, l_{2}\right)=\varphi\left(l_{1}, l_{2}\right) \bmod \boldsymbol{Z}$ is the geometric boundary $\partial(\varphi)$ of $\varphi$.

Now if $\varphi=\partial(\phi)$, and $\varphi$ is an even form with $p$ prime to $\operatorname{det}(\varphi)$, then our construction in $\S 2$ shows how to realize $\varphi$ by an action on a manifold-with-boundary $M$, having $\bar{\varphi}$ as the intersection form on $\partial M$. Of course, this is not always the case. We show, however, that up to equivalence one can find a form $\bar{\psi}$, Witt-equivalent to $\bar{\varphi}$, for which this is almost the case, and that is good enough. We proceed.

THeOREM 3.2. Let $\bar{\Phi}: \bar{L} \otimes \bar{L} \rightarrow Q / Z$ be a $\boldsymbol{Z}_{n}$-equivariant $\varepsilon$-symmetric bilinear form with $\bar{\varphi}=\partial(\varphi)$, where $\varphi: L \otimes L \rightarrow Z$ satisfies the conclusion of Theorem 1.6. Then there is a (k-1)-connected 2kmanifold $M$ with $(k-2)$-connected boundary $\partial M$, and a smooth $\boldsymbol{Z}_{p}$-action on $M$, free on $\partial M$, such that the linking form on $\partial M$ is Witt-equivalent to $\bar{\varphi}$. $k$ must be even if $\phi$ is symmetric and odd if $\phi$ is skew-symmetric; subject to that $k$ may be any integer $\geqq 3$.

Proof. If $W_{*}\left(\boldsymbol{Z}_{p}: Q\right)$ denotes the Witt group of $\boldsymbol{Z}_{p}$-equivariant $Q$-valued $\varepsilon$-symmetric bilinear forms (where $*=0$ for symmetric forms and $*=2$ for skew-symmetric forms), and similarly for $W_{*}\left(\boldsymbol{Z}_{p} ; \boldsymbol{Q} / \boldsymbol{Z}\right)$, then the geometric boundary map $\varphi \rightarrow \partial(\varphi)$ factors through the Witt groups to give a map $W_{*}\left(\boldsymbol{Z}_{p}: Q\right) \rightarrow \partial W_{*}\left(\boldsymbol{Z}_{p}: Q / Z\right)$. Furthermore, localization gives us a map

$$
0 \longrightarrow W_{*}\left(\boldsymbol{Z}_{p}: Q\right) \stackrel{\pi_{1}}{\oplus \pi_{2}} W_{*}\left(\boldsymbol{Z}_{p}: \boldsymbol{Z}(1 / p)\right) \oplus W_{*}\left(\boldsymbol{Z}_{p}: \boldsymbol{Z}(\mathbf{1} / \hat{p})\right)
$$

where $\boldsymbol{Z}(1 / \hat{p})$ is $\boldsymbol{Z}$ with all primes except $p$ inverted. Thus $\varphi \mapsto$ $\pi_{1}(\varphi) \oplus \pi_{2}(\varphi)$ where $\pi_{2}(\varphi)$ may be regarded as a bilinear form on a free $Z$-module whose determinant is a unit $\bmod p$. Thus if we can realize forms in the Witt classes of $\pi_{1}(\varphi)$ and $\pi_{2}(\varphi)$ we will be able to realize a form $\psi$ Witt-equivalent to $\varphi$ and hence with $\partial(\psi)$ Wittequivalent to $\partial(\varphi)$.

$W_{*}\left(\boldsymbol{Z}_{p}: \boldsymbol{Z}(\mathbf{1} / \hat{p})\right)$ is quite complex, but fortunately we need not concern ourselves with it. Let $\rho_{2}=\pi_{2}(\varphi)$, so $\rho_{2}: L_{2} \otimes L_{2} \rightarrow Z$. If $\rho_{2}$ is not itself an even form, we may replace it by $4 \rho_{2},\left(4 \rho_{2}\left(l_{1}, l_{2}\right)=\right.$ $\rho_{2}\left(2 l_{1}, 2 l_{2}\right)$, so we are merely rechoosing a lattice) which is Wittequivalent to $\rho_{2}$ (as $p$ is odd). Now since $\operatorname{det}\left(\rho_{2}\right)$ is a unit $\bmod p$, the constructions of $\S 2$ all go through and enable us to realize $\rho_{2}$. 
Now for $\rho_{1}=\pi_{1}(\varphi) \in W_{*}\left(Z_{p}: Z(1 / p)\right)$. Fortunately this group is relatively simple. There is a split exact sequence

$$
0 \longrightarrow W_{*}\left(\boldsymbol{Z}_{p}: \boldsymbol{Z}\right) \longrightarrow W_{*}\left(\boldsymbol{Z}_{p}: \boldsymbol{Z}(\mathbf{1} / p)\right) \stackrel{\partial}{\longrightarrow} W_{p}\left(\boldsymbol{Z}_{p}: \boldsymbol{Z}_{p}\right) \longrightarrow 0
$$

and $W_{2}\left(\boldsymbol{Z}_{p}: \boldsymbol{Z}_{p}\right)=0, W_{0}\left(\boldsymbol{Z}_{p}: \boldsymbol{Z}_{p}\right) \cong W\left(\boldsymbol{Z}_{p}\right)$.

We have already seen how to represent elements of $W_{*}\left(\boldsymbol{Z}_{p}: \boldsymbol{Z}\right)$ these are classes of $\boldsymbol{Z}_{p}$-equivariant, $\varepsilon$-symmetric, unimodular bilinear forms on free $\boldsymbol{Z}$-modules, so this is exactly the construction of $\S 2$.

It remains to represent $W_{0}\left(\boldsymbol{Z}_{p}: \boldsymbol{Z}_{p}\right) \cong W\left(\boldsymbol{Z}_{p}\right)$. Recall however, that $W\left(\boldsymbol{Z}_{p}\right)$ is given as follows:

$$
\begin{aligned}
& W\left(\boldsymbol{Z}_{p}\right)=\boldsymbol{Z}_{4} \text { generated by }\langle 1\rangle \text { for } p \equiv 3(4) \\
& W\left(\boldsymbol{Z}_{p}\right)=\boldsymbol{Z}_{2} \oplus \boldsymbol{Z}_{2} \text { generated by }\langle 1\rangle \text { and }\langle a\rangle, a \text { a quadratic } \\
& \text { nonresidue, for } p \equiv 3(4) \text {, where }\langle a\rangle \text { denotes the one- } \\
& \text { dimensional form }(x, y) \rightarrow a x y \text {. }
\end{aligned}
$$

But $\langle 1\rangle=\langle p+1\rangle$ and $\langle a\rangle=\langle a+p\rangle$ so we may always choose even representatives. Then an action realizing each of these is just given by an action on a boundle over a sphere of appropriate Euler class.

Thus we may always represent a form in the Witt class of $\rho_{1}$, and hence can always represent a form Witt-equivalent to $\bar{\varphi}$.

REMARK 3.3. It should be observed that in the case of torsion modules, taking the Witt class is an operation of extreme violence. For example, let $M_{n}=\boldsymbol{Z}_{p^{2}} \oplus \cdots \oplus \boldsymbol{Z}_{p^{2}}(n$ times $)$ provided with the $\boldsymbol{Z}_{p}$-action, whose matrix, with respect to the obvious basis, is

$$
\left(\begin{array}{ccccccc}
1 & & & & & \\
p & 1 & & & & \\
& p & 1 & & & \\
& p & 1 & & \\
& & & \ddots & \\
& & & & 1 & \\
& & & & p & 1
\end{array}\right)
$$

and whose $\boldsymbol{Z}_{p}$-valued bilinear form is $\sum_{i=1}^{n} x_{i}^{2}$. Then Heller and Reiner [5, I] show the $M_{n}, n=1,2, \cdots$, are all distinct indecomposable $\boldsymbol{Z}_{p^{-}}$ modules, yet the Witt class of each $M_{n}$ is trivial.

4. Locally smooth actions. Our constructions in $\S 2$ gave actions that were smooth everywhere except possibly at one fixed point, where the action was obtained by coning off the action on a homotopy 
sphere. The Atiyah-Singer G-Signature formula can be applied to decide when these actions can be made locally smooth (and sometimes smooth as well). Together with some number-theoretic results, this enables us to construct interesting examples of locally smooth actions with nonzero G-Signature.

First let us observe that we may modify the proof of Theorem 2.4 slightly. The representation at the fixed point of $M^{\prime}$ that we used was

$$
g\left(z_{1}, \cdots, z_{2 k}\right)=\left(\exp (2 \pi i / p) z_{1}, \cdots, \exp (2 \pi i / p) z_{2 k}\right) .
$$

In fact, we may use instead any representation

$$
g\left(z_{1}, \cdots, z_{2 k}\right)=\left(\exp \left(2 \pi i a_{1} / p\right) z_{1}, \cdots, \exp \left(2 \pi i a_{2 k} / p\right) z_{2 k}\right) .
$$

([9, Lemma 1.6] proves this in case $\prod_{i=1}^{k} a_{i}=\prod_{i=k+1}^{2 k} a_{i} \bmod p$ but it is in fact true in general.)

Now for any $2 k$-tuple $\left(a_{1}, \cdots, a_{2 k}\right)$, the proof of Lemmas 1.5 and 1.7 of $[10]$ show that

$$
\prod_{i=1}^{2 k} \cot \left(a_{i} \pi / p\right)=\frac{1}{p^{k}} \sum_{i=1}^{p-1} c_{i} \xi^{i}, \quad \xi=\exp (2 \pi i / p),
$$

with each $c_{i}$ an odd integer.

Fix a $2 k$-tuple $\left(a_{1}, \cdots, a_{2 k}\right)$ and let

$$
4 p^{k} \prod_{i=1}^{2 k} \cot \left(a_{i} \pi / p\right)=\sum_{i=1}^{p-1} d_{i} \xi^{i}
$$

Let us make the following assumption

Hypothesis (A): There exists an even, $Z_{p}$-equivariant, symmetric, unimodular bilinear form $\varphi: V \otimes V \rightarrow Z, V$ isomorphic to $m A$ for some $m$, with $\operatorname{Sign}(g, V)=\Sigma d_{i} \xi^{i}$.

Assuming this, we will construct locally smooth actions. Afterwards, we shall justify this assumption in some cases.

Let $\varphi$ be as in Hypothesis (A) and let $\psi=\varphi \oplus\left(m+4 p^{k}\right) \varepsilon$, where $\varepsilon$ is the two-dimensional hyperbolic plane with trivial $\boldsymbol{Z}_{p}$-action. Note $\operatorname{Sign}(g, \psi)=\operatorname{Sign}(g, \varphi)$

By Lemma 1.6 of [9] we know $4 p^{k} \varepsilon$ has a basis in which it has a matrix $H$ whose diagonal elements are mutually congruent, whose elements adjacent to the diagonal are congruent to 1 , and whose remaining elements are congruent to zero $\bmod p$.

Now perform the construction of 2.4 , so as to first obtain $\varphi \oplus$ $m \varepsilon$ as the form on a manifold-with-boundary having a $Z_{p}$-action with one fixed point, whose normal representation is

$$
g\left(z_{1}, \cdots, z_{2 k}\right)=\left(\exp \left(2 \pi a_{1} i / p\right) z_{1}, \cdots, \exp \left(2 \pi a_{2 k} i / p\right) z_{2 k}\right),
$$


and then, using $H$, continue the construction to obtain a manifoldwith-boundary $M^{\prime} . \quad M^{\prime}$ has a $Z_{p}$-action with $4 p^{k}+1$ isolated fixed points, each having the same normal representation.

Now on $\partial M^{\prime}$ we have a free $\boldsymbol{Z}_{p}$-action, whose quotient is a fake lens space $L$. We compute its multisignature from the $G$-Signature formula [1]

$$
\rho(L)=\operatorname{Sign}\left(g, M^{\prime}\right)-\sum_{i} \prod_{j=1}^{2 k} \cot \left(a_{j}^{i} \pi / p\right)
$$

where the sum is over all the fixed points, and the normal representation is $\left(a_{1}^{i}, \cdots, a_{2 k}^{i}\right)$ at the $i$ th fixed point. But here there are $4 p^{k}+1$ isolated fixed points, each with normal representation $\left(a_{1}, \cdots, a_{2 k}\right)$, while $\operatorname{Sign}\left(g, M^{\prime}\right)=\operatorname{Sign}(g, \varphi)$ by construction. Thus

$$
\begin{aligned}
\rho(L) & =\operatorname{Sign}(g, \varphi)-\left(4 p^{k}+1\right) \prod_{j=1}^{2 k} \cot \left(a_{j} \pi / p\right)=-\prod_{j=1}^{2 k} \cot \left(\alpha_{j} \pi / p\right) \\
& =\rho\left(L_{0}\right)
\end{aligned}
$$

by hypothesis $\mathrm{A}$, where $L_{0}$ is the linear lens space $L^{4 k-1}\left(p ;-a_{1}, a_{2}\right.$, $\left.\cdots, a_{2 k}\right)$. Then by [8, Chapter 14], $L$ is $P L h$-cobordant to $L_{0}$.

Adding in the universal cover of the $h$-cobordism, and then coning off the linear action covering the lens space $L_{0}$ gives a manifold $M$ with a locally smooth $\boldsymbol{Z}_{p}$-action having $G$-Signature $\Sigma d_{i} \xi^{i}$ (and having all fixed points isolated, with normal representations $\pm\left(a_{1}, \cdots, a_{2 k}\right)$.

Now we must investigate when hypothesis A is satisfied. We obtain a condition which is sufficient but undoubtedly not necessary. The argument is an adaptation for our purposes of a method of Pierre Conner's for construction unimodular forms, which we will outline.

Let $\mu \in \boldsymbol{Q}(\xi)$ be the unit $\xi^{(p-1) / 2}(1-\xi)$. Note $\bar{\mu}=-\mu$ (- denotes $^{-}$ complex conjugation).

Let $P$ be a projective $A$-module $(A=Z(\xi))$.

There is an isomorphism

$$
\operatorname{Hom}_{Z(\xi)}(P, Z(\xi)) \longrightarrow \operatorname{Hom}_{Z}(P, Z)
$$

given by $\psi \rightarrow 1 / p \operatorname{tr}(\mu \psi(x))$ where $\operatorname{tr}$ is the trace homomorphism in $\boldsymbol{Z}(\xi)$. (The $1 / p$ factor arises as $\mu \boldsymbol{Z}(\xi)$ is precisely the ideal of $\boldsymbol{Z}(\xi)$ of elements whose trace is divisible by $p$.)

Under this isomorphism a skew-Hermitian $Z(\xi)$-valued inner product $\langle$,$\rangle on p$ yields a symmetric even form $\beta$ on $P,(\beta\langle x, y\rangle=$ $1 / p \operatorname{tr}(\mu\langle x, y\rangle))$.

Consider a fractional ideal $I$ in $\boldsymbol{Q}(\xi)$. Let $k \in \boldsymbol{Q}\left(\xi+\xi^{-1}\right)$ be a unit for which $k I \bar{I}=\boldsymbol{Z}(\xi)$ ( $k$ is unique up to multiplication by a unit of $\left.\boldsymbol{Z}\left(\xi+\xi^{-1}\right)\right)$. 
We wish to introduce a skew-Hermitian $Z(\lambda)$-valued inner product on $P=\boldsymbol{Z}(\xi) \oplus I \subset \boldsymbol{Q}(\xi) \oplus \boldsymbol{Q}(\xi)$. This will be given by

$$
\langle(x, y),(v, w)\rangle=(x, y) S^{t}(\overline{v, w})
$$

for a suitable matrix $S$.

$\mathrm{S}$ must be skew-Hermitian (with entries in $\boldsymbol{Q}(\xi)$ ) and $S$ must be $\boldsymbol{Z}(\xi)$-valued on $\boldsymbol{Z}(\xi) \oplus I$. If $S=\left(\begin{array}{rr}\alpha & \beta \\ -\beta & \gamma\end{array}\right)$ this will occur exactly when $\bar{\alpha}=-\alpha, \bar{\gamma}=-\gamma, \alpha \in \boldsymbol{Z}(\xi), \beta \in I^{-1}$, and $\gamma \in k Z(\xi)$. In such case we may rewrite $S$ in the form

$$
S=\left(\begin{array}{cc}
\mu \alpha & \beta \\
-\bar{\beta} & k \mu \gamma
\end{array}\right), \quad \alpha, \gamma \in \boldsymbol{Z}\left(\xi+\xi^{-1}\right), \quad \beta \in I^{-1} .
$$

In order for $S$ to be an inner product we also need $\operatorname{det}(S)=k u$ for some unit $u$ of $\boldsymbol{Z}\left(\xi+\xi^{-1}\right)$, giving the condition

$$
\mu^{2} \alpha \gamma+k^{-1} \beta \bar{\beta}=u \text {. }
$$

We will call such a unit $u$ adapted to the pair $(I, k)$.

The field $\boldsymbol{Q}\left(\xi+\xi^{-1}\right)$ has $(p-1) / 2$ different orderings corresponding to the $(p-1) / 2$ embeddings of $\boldsymbol{Q}(\xi)$ into $C$ (conjugate embeddings give the same ordering and so are excluded), and it turns out that $u$ is adapted to $(I, k)$ if and only if $u k$ is negative at an even number of orderings. Now pairs $(I, k)$ exist where the sign of $k$ at each ordering may be arbitrarily prescribed.

Thus if an even set of orderings is chosen, there is a pair $(I, k)$ to which 1 is adapted and for which $k$ is negative at exactly those orderings.

We may now calculate the G-Signature of the form arising from such a matrix $S$ with $\alpha=1$. Since $\beta(x, y)=1 / p \operatorname{tr}(\mu\langle x, y\rangle)$ we multiply $S$ by $\mu$ to obtain the Hermitian matrix

$$
\left(\begin{array}{cc}
\mu^{2} & \mu \beta \\
-\mu \bar{\beta} & k \mu^{2} \gamma
\end{array}\right)
$$

If $T$ is the unimodular matrix $\left(\begin{array}{cc}1 & 0 \\ \bar{\beta} / \mu & 1\end{array}\right)$, conjugating $S$ by $T$ gives $T S T^{*}=\left(\begin{array}{ll}\mu^{2} & 0 \\ 0 & \beta \bar{\beta}-k \mu^{2} \gamma\end{array}\right)=\left(\begin{array}{ll}\mu^{2} & 0 \\ 0 & k\end{array}\right)$.

Now $\mu^{2}$ is negative at every ordering of $\boldsymbol{Q}\left(\xi+\xi^{-1}\right)$, so at the orderings of $\boldsymbol{Q}\left(\xi+\xi^{-1}\right)$ where $k$ is negative we get a contribution of -2 to the total signature, while at the others we get a contribution of 0 . Changing signs gives us contributions of +2 or 0 .

Thus we can realize $G$-Signatures which occur as sums $\Sigma\left(\Sigma \alpha_{i} \xi^{i}\right)$ with $a_{i}= \pm 2$ or 0,2 or -2 occurring an even number of times, so 
certainly the sum $\Sigma d_{i} \xi^{i}$ of $\left(^{*}\right)$ can be realized as each $d_{i}$ is divisible by four.

Thus we see that we can find a form $\varphi$ on $\oplus(\boldsymbol{Z}(\xi) \oplus I)$ with $\operatorname{Sign}(g, \varphi)=\Sigma d_{i} \xi^{i}$. The only remaining point is that we need to ensure that $I$ is actually a principal ideal. Actually, we do not need quite this strong a result, it suffices that our form $\phi$ should be Witt-equivalent to a form $\varphi^{\prime}$ whose underlying space is $\oplus \boldsymbol{Z}(\xi)$.

Now the ideal $I$ defines a class in $H_{2}\left(\boldsymbol{Z}_{2}, \mathscr{C}\right)$, with $\mathscr{C}$ the ideal class group of $\boldsymbol{Z}(\xi)$, and $\boldsymbol{Z}_{2}$ acting on an ideal by conjugation, and $\varphi$ is Witt-equivalent to such a form $\phi^{\prime}$ iff this class is zero. In general, it is difficult to decide when $I$ is zero, but if the order of $\mathscr{C}$ is odd, $H_{2}\left(Z_{2}, \mathscr{C}\right)=0$ and so the class of $I$ must trivially be zero.

Thus, we may conclude that hypothesis (A) is satisfied if the class number of $\boldsymbol{Q}(\xi)$ is odd.

Summarizing we have shown

EXAMPLE 4.1. Let $p$ be a prime with the class number of $\boldsymbol{Q}(\exp (2 \pi i / p))$ odd. Let $k \geqq 2$ and let $\left(a_{1}, \cdots, a_{2 k}\right)$ be a $2 k$-tuple of integers prime to $p$.

Let $\varphi$ be any $\boldsymbol{Z}_{p}$-equivariant even integer-valued unimodular form with $\boldsymbol{Z}_{p}$.

$\operatorname{Sign}(g, \varphi)=$ any multiple of $4 p^{k} \prod_{i=1}^{2 k} \cot \left(a_{i} \pi / p\right), g$ a generator of

Such a form $\varnothing$ always exists.

Then there is a $(2 k-1)$-connected $4 k$-manifold $M$ admitting a semi-free locally smooth $\boldsymbol{Z}_{p}$-action, with isolated fixed points whose local representation are all $g\left(z_{1}, \cdots, z_{k}\right)=\left(\exp \left( \pm 2 \pi i a_{1} / p\right) z_{1}\right.$, $\left.\exp \left(2 \pi i a_{2} / p\right) z_{2}, \cdots, \exp \left(2 \pi i a_{2 k} / p\right) z_{2 k}\right)$, and with $\varphi$ the equivariant intersection form $\varphi: H_{2 k}(M) \otimes H_{2 k}(M) \rightarrow Z$.

\section{REFERENCES}

1. M. F. Atiyah, and I. M. Singer, The index of elliptic operators III, Ann. of Math.,

(2) 87 (1968), 546-604.

2. G. Bredon, Introduction to Compact Transformation Groups, Academic Press, New York-London, 1972.

3. W. Browder, Surgery on Simply-Connected Manifolds, Springer-Verlag, BerlinHeidelberg-New York, 1972.

4. C. Curtis and I. Reiner, Representation Theory of Finite Groups and Associative Algebras, John Wiley and Sons, New York-London, 1962.

5. A. Heller and I. Reiner, Representations of cyclic groups in rings of integers I and II, Ann. of Math., (2) 76 (1962), 73-92 and 77 (1963), 318-328.

6. T. Lance, Free cyclic actions on manifolds, Comm. Math. Helv., 50 (1975), 59-80.

7. R. Swan, The Grothendieck ring of a finite group, Topology, 2 (1963), 85-110.

8. C. T. C. Wall, Surgery on Compact Manifolds, Academic Press, New York-London, 1970. 


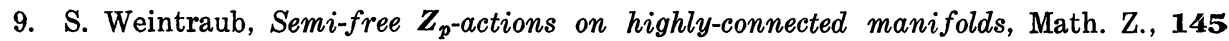
(1975), 163-185.

10. , $Z_{p}$-actions and the rank of $H_{n}\left(N^{2 n}\right)$, J. London Math. Soc., 13 (1976), $565-572$.

Received March 15, 1976 and in revised form November 19, 1976. Partially supported by NSF Grant MPS 74-07563 and an LSU Summer Faculty Research Appointment. Louisiana State University

BATON ROUGE, LA 70803 


\section{PACIFIC JOURNAL OF MATHEMATICS}

EDITORS

RICHARD ARENS (Managing Editor)

University of California

Los Angeles, California 90024

C. W. Curtis

University of Oregon

Eugene, OR 97403

C. C. MOORE

J. DUGUNDJI

Department of Mathematics University of Southern Californa Los Angeles, California 90007

R. Finn AND J. Milgram Stanford University Stanford, California 94305

University of California

Berkeley, CA 94720

\section{ASSOCIATE EDITORS}

E. F. BeCKENBACH

B. H. NeUMANN

F. WOLF

K. YosHida

\section{SUPPORTING INSTITUTIONS}

UNIVERSITY OF BRITISH COLUMBIA UNIVERSITY OF SOUTHERN CALIFORNIA CALIFORNIA INSTITUTE OF TECHNOLOGY UNIVERSITY OF CALIFORNIA MONTANA STATE UNIVERSITY UNIVERSITY OF NEVADA, RENO STANFORD UNIVERSITY UNIVERSITY OF TOKYO UNIVERSITY OF UTAH NEW MEXICO STATE UNIVERSITY WASHINGTON STATE UNIVERSITY OREGON STATE UNIVERSITY UNIVERSITY OF WASHINGTON UNIVERSITY OF OREGON OSAKA UNIVERSITY 


\section{Pacific Journal of Mathematics \\ Vol. 73, No. 1 \\ March, 1977}

Thomas Robert Berger, Hall-Higman type theorems. $V \ldots \ldots \ldots \ldots \ldots \ldots \ldots$

Frank Peter Anthony Cass and Billy E. Rhoades, Mercerian theorems via

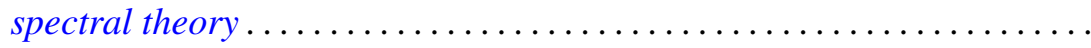

Morris Leroy Eaton and Michael David Perlman, Generating $\mathrm{O}(n)$ with

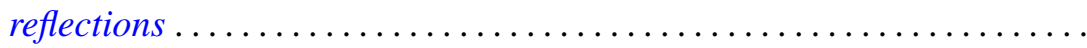

Frank John Forelli, Jr., A necessary condition on the extreme points of a

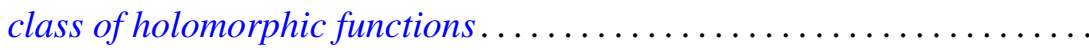

Melvin F. Janowitz, Complemented congruences on complemented

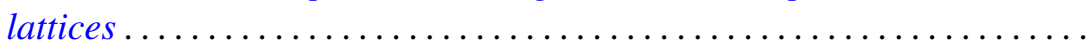

Maria M. Klawe, Semidirect product of semigroups in relation to amenability, cancellation properties, and strong $F \phi$ lner conditions....

Theodore Willis Laetsch, Normal cones, barrier cones, and the "spherical image" of convex surfaces in locally convex spaces ................

Chao-Chu Liang, Involutions fixing codimension two knots.............

Joyce Longman, On generalizations of alternative algebras .............

Giancarlo Mauceri, Square integrable representations and the Fourier

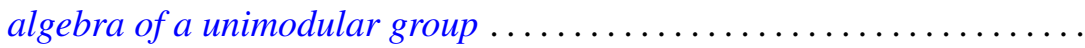

J. Marshall Osborn, Lie algebras with descending chain condition...

John Robert Quine, Jr., Tangent winding numbers and branched

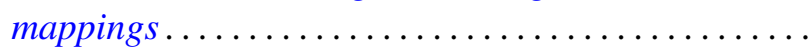

Louis Jackson Ratliff, Jr. and David Eugene Rush, Notes on ideal covers and associated primes .

H. B. Reiter and N. Stavrakas, On the compactness of the hyperspace of faces.

Walter Roth, A general Rudin-Carlson theorem in Banach-spaces ..

Mark Andrew Smith, Products of Banach spaces that are uniformly rotund in every direction.

Roger R. Smith, The R-Borel structure on a Choquet simplex ...

Gerald Stoller, The convergence-preserving rearrangements of real infinite series. ...

Graham H. Toomer, Generalized homotopy excision theorems modulo a Serre class of nilpotent groups...

Norris Freeman Weaver, Dehn's construction and the Poincaré conjecture....

Steven Howard Weintraub, Topological realization of equivariant intersection forms... 